Relations industrielles

Industrial Relations

\title{
Penrose, Roger, Shadows of the Mind: A Search for the Missing Science of Consciousness
}

\section{Rolland Hurtubise}

Volume 50, numéro 3, 1995

URI : https://id.erudit.org/iderudit/051048ar

DOI : https://doi.org/10.7202/051048ar

Aller au sommaire du numéro

Éditeur(s)

Département des relations industrielles de l'Université Laval

ISSN

0034-379X (imprimé)

1703-8138 (numérique)

Découvrir la revue

Citer ce compte rendu

Hurtubise, R. (1995). Compte rendu de [Penrose, Roger, Shadows of the Mind: A Search for the Missing Science of Consciousness]. Relations industrielles /

Industrial Relations, 50(3), 677-680. https://doi.org/10.7202/051048ar

Tous droits réservés @ C Département des relations industrielles de l'Université Laval, 1995
Ce document est protégé par la loi sur le droit d'auteur. L'utilisation des services d'Érudit (y compris la reproduction) est assujettie à sa politique d'utilisation que vous pouvez consulter en ligne.

https://apropos.erudit.org/fr/usagers/politique-dutilisation/ 
tributed to the empirical knowledge as to the actual effects of standards on the process and economic outcome of restructuring? Of the two objectives, I would say that they were more successful in accomplishing the second objective. However, there is no question that this book should prove useful to those who are looking for both the evidence and the rationale for a greater role of the state in the intelligent use of labour standards as a means to channel market forces toward socially desirable directions.

MAURICE MAZEROLLE Toronto, Ontario

\section{Shadows of the Mind: A Search for the Missing Science of Consciousness} by Roger PENROSE, Oxford, Angleterre, Oxford University Press, 1994, xvi+457 p. ISBN 0-19-853978-9.

\section{La conscience chez l'ordinateur}

L'ordinateur aura-til une conscience? Pourra-t-il penser? Sera-t-il intelligent? Bref, le domaine de l'intelligence artificielle (IA) a-t-il un avenir? Le récent livre du physicien anglais Roger Penrose communique un message qui est le prolongement de son précédent, L'esprit, l'ordinateur et les lois de la physique (Paris, InterÉditions, 1992), dans lequel il maintenait que jamais un ordinateur ne sera conscient, donc jamais véritablement intelligent. Dans Shadows of the Mind. A Search for the Missing Science of Consciousness, l'auteur explique que notre "mentalité consciente " et ses diverses manifestations ne seront jamais comprises par des modèles fondés sur des calculs.

\section{À la recherche d'une physique nouvelle}

Il y a deux parties au récent livre. À la partie I (trois chapitres), Penrose réfère à un "ingrédient " absent de la façon de voir les choses scientifiquement. Selon lui, il n'existe pas encore de théories physique, biologique et de calcul qui permettent d'expliquer notre conscience et, par conséquent, notre intelligence. L'ordinateur de l'avenir aurat-il un esprit? En guise de réponse à ce genre de questions, l'auteur propose, dès le premier chapitre, quatre options (p. 12):

A- Toute pensée est fondée sur le calcul ; les sentiments d'être conscient sont évoqués simplement en réalisant les calculs appropriés.

B- Etre conscient est une caractéristique de l'action physique du cerveau; alors que toute action physique peut être simulée par des calculs, la simulation par calculs seule ne peut pas rendre conscient.

C- L'action appropriée du cerveau rend conscient, mais cette action physique ne peut même pas être convenablement simulée par des calculs.

D- Etre conscient ne s'explique pas en des termes physiques, ni de calculs, ni en d'autres termes scientifiques.

L'option A est celle des défenseurs d'une IA qualifiée de "forte". Ceux-ci maintiennent qu'il est possible de simuler la conscience en ordinateur par des calculs et alors le rendre conscient. L'option B est celle de l'IA qualifiée de "faible". Si ses partisans reconnaissent la possibilité de simuler par des calculs la conscience, ils conviennent que l'ordinateur en cause ne sera pas conscient pour autant. Quant à l'option D, elle est celle du mysticisme, des doctrines religieuses. L'auteur démolit ces trois options et retient l'option $\mathrm{C}$ où il reconnaît que les questions reliées à l'esprit ne doivent pas se situer éternellement à l'extérieur des domaines scientifiques. Il signale qu'un atout de l'option C est son ouverture sur de futures réalisations scientifiques qui permettraient la construction d'appareils fondés sur des actions 
physiques non calculées reliées à la pensée, à l'intelligence, à la conscience (p. 35).

\section{Des définitions plus précises?}

L'auteur a été critiqué pour ne pas avoir avancé des définitions plus précises de certains substantifs, dont " conscience" et "esprit " (A. Sloman, "The Emperor's Real Mind: Review of Roger Penrose's The Emperor's New-Mind: Concerning Computers, Minds and the Law of Physics ", Artificial Intelligence, № 56, 1992, 355-396). Même s'il affirme être "souvent décontenancé" par ce genre de critiques, il persiste à croire que des définitions complètes seraient inutiles (p. 37). Néanmoins, le lecteur découvre une progression soutenant que l'intelligence exige la compréhension qui, par ricochet, demande d'être conscient. Quant à la notion d'être conscient, elle constituerait le volet passif du phénomène de la conscience, tandis que son volet actif serait celui du libre arbitre (free will).

\section{Des preuves}

C'est au chapitre 2 que Penrose offre l'argumentation en faveur de cet "ingrédient non calculé propre à la pensée consciente ". Il réfère et expose longuement le théorème de Kurt Gödel, le mathématicien américain qui a démontré qu'une arithmétique non contradictoire ne saurait former un système complet, car la non-contradiction y constitue un énoncé indécidable. Par l'entremise de ce théorème illustré à l'aide du fonctionnement de la machine à Turing, cet ordinateur mathématiquement idéalisé, l'auteur explique que la compréhension humaine n'est pas une activité algorithmique, que l'intuition et la perspicacité ne peuvent pas être réduites à un ensemble de règles (p. 65).

Au chapitre 3, l'auteur fait valoir qu'un "robot " ne possédera jamais les aptitudes d'un mathématicien humain, car celui-ci possède un concept de "vé- rité mathématique irréfutable" qui ne peut pas être transmuté en un ensemble de règles mécaniques (connaissable et entièrement croyable par l'humain) (p. 158). Par une argumentation de type reductio ad absurdum, il déclare non seulement que le robot est incapable de savoir qu'il a été construit selon un mécanisme quelconque, mais que des mécanismes qui sauraient lui inculquer un raisonnement mathématique correct ne peuvent pas exister ou ne peuvent pas être connus ni du robot, ni (par conséquent) de l'humain (p. 195).

\section{Une physique nouvelle pour compren-} dre l'esprit

Aux cing chapitres de la partie II, l'auteur est à la recherche d'une physique sans calcul qui permettrait de comprendre l'esprit. Au chapitre 4, il ne discute pas directement de l'incalculabilité, mais particulièrement de la théorie de la relativité générale d'Einstein et de l'inclinaison des cônes lumineux due à la gravité. Penrose précise que rien de connu en physique sauf la gravité peut incliner des cônes lumineux (p. 225). Pourquoi se sert-il du sujet de l'effet du fléchissement de la lumière par l'attraction gravitationnelle? Parce qu'elle offre une morale! "il est très possible, en physique, d'avoir une nouvelle propriété, foncièrement importante, complètement différente des précédentes, cachée et inobservée dans le comportement de la matière ordinaire. [...] Par analogie, nous pouvons envisager l'existence d'une action sans calcul cachée quelque part dans le comportement des choses" (p.227). (Le lecteur intéressé trouvera aux chapitres 5 et 6 un cours sur la théorie quantique.)

Le chapitre 7, intitulé "La théorie quantique et le cerveau ", est primordial. L'auteur remarque que la physique classique n'a pas l'étrange "superpositionnement " de la théorie quantique où l'on admet qu'un événement peut avoir lieu et ne pas avoir lieu simultanément (p. 348). Il exprime aussi qu'une action 
non calculée doit survenir à cause d'un phénomène cohérent quantique à grande échelle qui, discrètement couplé au comportement macroscopique, rend le système en cause apte à profiter de nouveaux processus physiques. Penrose es time que même si les signaux neuronaux se comportent classiquement, les liens synaptiques entre eux sont contrôlés à un niveau plus profond, là où survient une activité physique à la frontière qui sépare le quantique du classique. Mais où se situe exactement le comportement cohérent quantique à grande échelle? ¿̀ l'intérieur des microtubules (tubes cylindriques) des cytosquelettes (les charpentes internes) des neurones (p. 357)! Roger Penrose croit que les microtubules - responsables de la force synaptique et de la croissance des liens entre les neurones - contrôlent l'activité du cerveau et que leur action est différente du simple calcul (p. 367). Plutôt que les neurones, ce sont les microtubules et leurs effets quantiques qui offrent la possibilité de cerner scientifiquement le phénomène de la conscience.

C'est vers la fin du chapitre 7 que la signification du titre du livre, Les ombres de l'esprit, est soumise au lecteur: "la description de niveau neuronal qui offre le portrait à la mode du cerveau et de l'esprit n'est qu'une ombre du niveau plus profond de l'action cytosquelettique - et c'est à ce niveau plus profond qu'il faut rechercher la base physique de l'esprit " (p. 376).

\section{Réunir les nombreux thèmes}

Au chapitre 8, Penrose rappelle qu'une machine intelligente - en mesure de comprendre - ne sera jamais fondée sur la technologie informatique telle que nous la connaissons, mais que sa fabrication devra tenir compte de l'option $\mathrm{C}$ qui permet à la conscience, à l'intelligence, à l'esprit d'être compris en des termes scientifiques mais dépourvus de calcul (p. 393). En outre, ce chapitre contient une tentative de réunir les thèmes du livre. L'auteur présente trois "mondes", le monde platonique des formes mathématiques, le monde physique et le monde de la perception consciente. Ceux-ci sont reliés de façon telle qu'une petite partie du premier est projetée sur la totalitê du second, une petite partie du second est projetée sur la totalité du troisième et, pour boucler la boucle, une petite partie du troisième est projetée sur la totalité du premier. Trois mystères les accompagnent: des lois mathématiques précises et profondes jouant un rôle important sur le comportement du monde physique, des êtres perceptifs (mentalement) provenant du monde physique et l'état mental créant des concepts mathématiques (p. 414). L'auteur admet que certains peuvent mettre en doute la séquence des "mondes": lequel est à l'ombre de l'autre?

\section{Appréciation}

Toute personne intéressée de près ou de loin par la pensée, l'intelligence, la conscience et d'autres propriétés de l'esprit chez l'ordinateur doit lire Les ombres de l'esprit. (Serait-ce le titre français ?) Le lecteur qui aura l'occasion de comparer ce livre au précédent ressentira que l'auteur a voulu répondre à ses détracteurs, aussi rapidement que possible. Néanmoins, il faut admirer son honnêteté intellectuelle. Il avertit que certains points de vue sont les siens et qu'il ne sera pas très équitable envers les convictions des autres. Il s'en excuse. Il indique aussi qu'il est possible qu'il n'ait pas raison (p. 316).

Ce ne sont pas les descriptions ni les explications reliées aux principes et théories pris individuellement qu'il faut chercher à mettre en cause. Là où le bât blesse se situe au niveau de leur emploi pour expliquer des phénomènes et des concepts peu, mal ou pas définis. Comment peut-on entamer de sérieuses discussions scientifiques (mathématiques) 
si les termes conscience, être conscient, compréhension, esprit, pensée, penser et intelligence n'ont pas été préalablement élucidés? Il est difficile de concilier les arguments hautement mathématiques et précis avec les notions floues de l'esprit. Peut-être que la délimitation de l'emploi de certains mots aurait diminué des confusions. À titre d'exemple, lorsqu'il est question des compréhensions mathématiques, qu'en est-il des compréhensions non mathématiques? Ces dernières existent-elles? Si oui, sont-elles vraiment identiques aux formes mathématiques? Penrose lui-même admet que l'ar- gumentation de Gödel "oblige une attitude rationnelle serrée à l'égard de ses croyances mathématiques irréfutables, alors que le comportement humain courant est rarement de genre rationnel aussi précis " (p. 207). Si l'ordinateur ne peut pas penser comme un mathématicien, pourra-til penser comme un nonmathématicien? Voilà la question.

\section{ROLIAND HURTUBISE}

École nationale d'administration publique

\section{Association canadienne des relations industrielles (ACRI) Canadian Industrial Relations Association (CIRA)}

L'ACRI est une organisation volontaire à but non lucratif. Fondée en 1963, elle a pour objectif la promotion de la recherche, les discussions et l'éducation dans le domaine des relations industrielles au Canada. Les membres se recrutent dans les milieux syndicaux, patronaux, gouvernementaux, chez les arbitres, les enseignants, les chercheurs, les conseillers et autres spécialistes de relations industrielles. LACRI organise son congrès annuel dans le cadre des conférences des Sociétés savantes du Canada. La cotisation annuelle à l'ACRI inclut l'abonnement à la revue Relations industrielles/Industrial Relations, organe officiel de l'ACRI.

CIRA is a non-profit voluntary organization founded in 1963 and devoted to the promotion of research, discussion and education in the field of industrial relations in Canada. CIRA's membersbip includes people from unions, management and government interested in industrial relations as well as arbitrators, researchers, teacbers, consultants and other specialists in the field. Its annual conference is jointly organized with the Learned Societies of Canada. CIRA's annual dues include a year's subscription to Relations industrielles/Industrial Relations, its official organ.

Secrétariat/Secretariat - ANTHONY GILES

Département des relations industrielles, Pavillon J.-A.-De Sève

Université Laval, Québec, Canada, G1K 7P4

Tél. (418) 656-2514/2468 - Fax (418) 656-3175 